\title{
Resequencing microarray probe design for typing genetically diverse viruses: human rhinoviruses and enteroviruses Zheng Wang* ${ }^{\dagger 1}$, Anthony P Malanoski ${ }^{\dagger 1}$, Baochuan Lin ${ }^{1}$, Carolyn Kidd ${ }^{2}$, Nina C Long2, Kate M Blaney ${ }^{2}$, Dzung C Thach ${ }^{1}$, Clark Tibbetts ${ }^{3}$ and David A Stenger ${ }^{1}$
}

\author{
Address: ${ }^{1}$ Center for Bio/Molecular Science \& Engineering, Naval Research Laboratory, Washington, DC 20375, USA, ${ }^{2}$ Nova Research Inc, \\ Alexandria, VA 22308, USA and ${ }^{3}$ Tessarae, LLC, Potomac Falls, VA 20165, USA \\ Email: Zheng Wang* - zheng.wang@nrl.navy.mil; Anthony P Malanoski - anthony.malanoski@nrl.navy.mil; \\ Baochuan Lin - baochuan.lin@nrl.navy.mil; Carolyn Kidd - caro_kidd@verizon.net; Nina C Long - nina.long@nrl.navy.mil; \\ Kate M Blaney - kate_m_mueller@yahoo.com; Dzung C Thach - dzung.thach@nrl.navy.mil; Clark Tibbetts - clark.tibbetts@tessarae.com; \\ David A Stenger - david.stenger@nrl.navy.mil \\ * Corresponding author †Equal contributors
}

Published: I December 2008

BMC Genomics 2008, 9:577 doi:10.1/86/147|-2164-9-577

This article is available from: http://www.biomedcentral.com/I47/-2/64/9/577

(C) 2008 Wang et al; licensee BioMed Central Ltd.

This is an Open Access article distributed under the terms of the Creative Commons Attribution License (http://creativecommons.org/licenses/by/2.0), which permits unrestricted use, distribution, and reproduction in any medium, provided the original work is properly cited.
Received: 28 April 2008

Accepted: I December 2008

\begin{abstract}
Background: Febrile respiratory illness (FRI) has a high impact on public health and global economics and poses a difficult challenge for differential diagnosis. A particular issue is the detection of genetically diverse pathogens, i.e. human rhinoviruses (HRV) and enteroviruses (HEV) which are frequent causes of FRI. Resequencing Pathogen Microarray technology has demonstrated potential for differential diagnosis of several respiratory pathogens simultaneously, but a high confidence design method to select probes for genetically diverse viruses is lacking.
\end{abstract}

Results: Using HRV and HEV as test cases, we assess a general design strategy for detecting and serotyping genetically diverse viruses. A minimal number of probe sequences ( 26 for HRV and 13 for HEV), which were potentially capable of detecting all serotypes of HRV and HEV, were determined and implemented on the Resequencing Pathogen Microarray RPM-Flu v.30/3I (Tessarae RPM-Flu). The specificities of designed probes were validated using $34 \mathrm{HRV}$ and $28 \mathrm{HEV}$ strains. All strains were successfully detected and identified at least to species level. 33 HRV strains and 16 HEV strains could be further differentiated to serotype level.

Conclusion: This study provides a fundamental evaluation of simultaneous detection and differential identification of genetically diverse RNA viruses with a minimal number of prototype sequences. The results demonstrated that the newly designed RPM-Flu v.30/3I can provide comprehensive and specific analysis of HRV and HEV samples which implicates that this design strategy will be applicable for other genetically diverse viruses.

\section{Background}

Human febrile respiratory illness (FRI) results in significant annual health and economic burden worldwide, but the diversity and number of pathogens make differential diagnosis very challenging. Thus, it represents a useful example where many organisms ranging from bacteria 
(Haemophilus influenzae) to fairly conserved viruses (respiratory syncytial virus) to genetically diverse viruses, i.e. influenza A virus, human rhinoviruses (HRV), and human enteroviruses (HEV) need to be detected for successful differential diagnosis. Several technologies, Mass$\operatorname{code}^{\mathrm{TM}}$ multiplex RT-PCR system [1], electrospray ionization mass spectrometry analysis of PCR amplicons [2], Luminex $\mathrm{xMAP}^{\mathrm{TM}}[3]$, and various microarray-based approaches [4-8], are currently under development as diagnostic platforms to effectively and simultaneously detect and identify large numbers of diverse viral and bacterial respiratory pathogens. One high-density resequencing microarray platform, the Respiratory Pathogen Microarray version 1 (RPM v.1), has been successfully demonstrated to identify a much broader range of pathogens (including bacteria and DNA and RNA viruses) in a single test at sensitivities and specificities that are similar to or improved over those of other technologies $[9,10]$. In addition, the RPM v.1 platform has the demonstrated capability to discriminate among known and previously unknown strains and variants of targeted pathogens $[11,12]$.

While promising, the RPM v.1 platform was a proof-ofconcept microarray for the detection of 26 common respiratory pathogens primarily encountered among military basic trainees. It did not provide comprehensive coverage of all potential respiratory pathogens and the design methodology used was not appropriate for genetically diverse viruses. The design methodology for the RPM v.1 microarray consisted of applying selection rules developed for long oligonucleotide microarrays. These rules were not optimal but worked for bacterial organisms and fairly conserved viruses since previous studies had shown a single sequence on a resequencing microarray could reliably detect and serotype strains with as much as 10 to $15 \%$ variation $[8,10-12]$. Their application to cover more diverse viral organisms was less successful. For example, the $5^{\prime}$ untranslated region (5'UTR) sequence chosen for HRV on the RPM v.1 only provided identification of the prototype HRV-89 and very little coverage of other HRV serotypes. The 5'UTR sequences, which are relatively conserved among HRV and HEV, have been used in PCR and de novo sequencing for tentative viral identification or serotype classification in lieu of the much more variable capsid proteins that actually determine serotypes $[13,14]$. However, the 5'UTR sequences still have 5 to $30 \%$ nucleotide sequence variations among different serotypes so require more than one prototype sequence for proper identification and serotyping. Serotyping HRV and HEV is important to FRI differential diagnosis because even though these "common cold" viruses generally only induce mild symptoms, they can cause a wide variety of other severe illnesses, such as aseptic meningitis [15], bronchitis and asthma [16].
New resequencing pathogen microarray designs, versions 3.0 and 3.1 (RPM-Flu v.30/31), have been constructed to address the shortcomings of the previous design. The use of $8 \mu \mathrm{m}$ feature allows microarrays with greater coverage, currently 86 , of common respiratory organisms and high human health risk zoonotic pathogens (bacteria and viruses). A new approach to select a minimal number of prototype sequences that can be used to detect all and correctly identify many of the relevant strains of genetically diverse viruses such as HRV and HEV was developed. Due to the great genetic diversity of HRV and HEV, in order to ensure that designed probes (referred to as probe sequences) generated from selected database sequences (referred to as prototype regions) would detect and discriminate all serotypes of HRV and HEV, a predictive model was used to assist the microarray design [17]. This in silico model developed for predicting resequencing microarray hybridization patterns shows good concordance in the overall percentage of base calls predicted versus experimental results. Thus it is possible to use this model for evaluating the performance of database sequences as potential prototype regions. In this study, we report on results of this algorithm applied to the 5'UTR sequences of HRV and HEV and confirm that using 15\% of the RPM-Flu v.30/31 microarray $(17,335 \mathrm{HRV}$ and HEV nucleotides of total 117,254 nucleotides on array) is sufficient to detect and differentiate many HRV and HEV serotypes.

\section{Results \\ Microarray design process \\ In silico modeling}

Figure 1 illustrates the procedures used for the selection of HRV and HEV probe sequences. First, sequences that contain the specified target region (5'UTR) and meet any selection criteria applied were downloaded from a database (currently GenBank). Downloaded sequences were trimmed to cover the same region using pair-wise sequence alignment. These sequences were treated as target sequences (what would be detected by the microarray) and also as prototype sequences (the potential probe sequences tiled on the microarray). Each downloaded sequence was treated as a prototype used to generate probe sequences (Fig 1. Step 1-2) and the remaining sequences were treated as target sequences (Fig 1. Step 34 ). Sets of 4 25-mer probes (1 perfect match and 3 mismatches in the $13^{\text {th }}$ position) were generated from a prototype sequence and correspond to what would actually appear on a resequencing microarray. The other sequences were treated as a target one at a time and generated overlapping fragments from 13 to 25 bases long with a near neighbor $\Delta \mathrm{G}$ energy less than -14.5. These fragments have been shown to have strong binding strength and produce unique base calls. The generated probes and sequence fragments were the input to the in silico model 


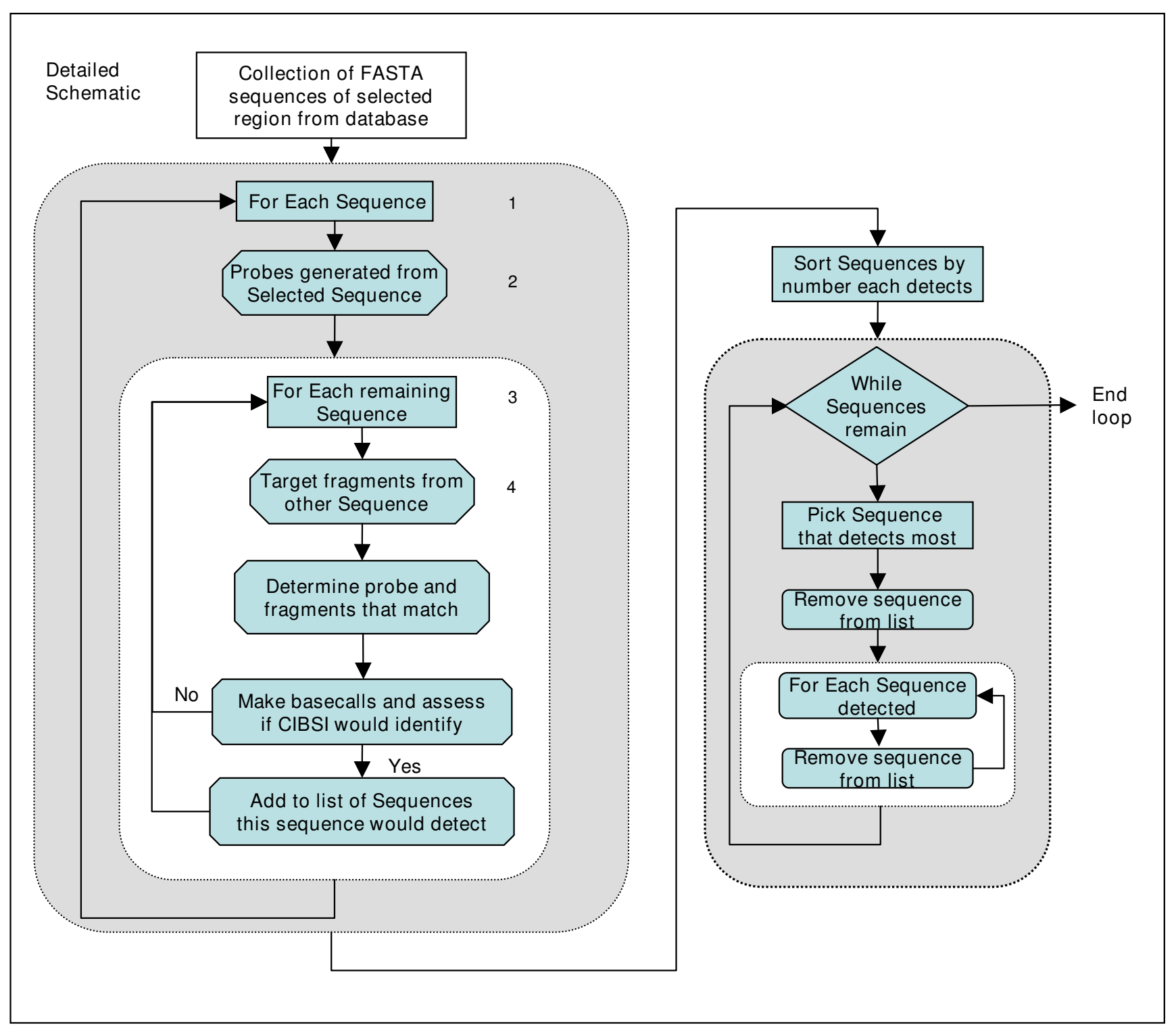

Figure I

Schematic of algorithm representing the prototype sequences selection process. A collection of database sequences covering a specified region are processed together. Each sequence is treated as probe sequence that the other sequences are tested against. The numbers of these sequences detected by the probe sequences are determined. A group of sequences that are predicted to detect all the sequences is then selected.

[17] for simulation which compared the fragments to the probe sets and determined the base calls a target sequence would generate. The predicted base calls were assembled into a simulated resequencing microarray result.

\section{Probe sequence selection}

The simulated result of a target sequence for the current prototype sequence was then run through the previously developed CIBSI analysis algorithm [8] with the following criteria. A sequence was considered detected by the cur- rent prototype sequence if at least one region of 50 or more contiguous nucleotides was predicted to consist of A, C, G, and T base calls and no ambiguous base calls (Ns). As shown in Figure 1 "Yes" for "CIBSI would identify" updated the list of sequences that could be detected by the current prototype sequence. This procedure was applied for every downloaded sequence. For example, if we collect sequences " $a-z$ " from GenBank, we will first use sequence " $a$ " as a prototype sequence, then use sequences "b-z" each in turn to generate target sequences for in silico 
simulation. After the completion of the simulation and CIBSI analysis, a list of sequences from the pool of sequences "b-z" that can be detected by prototype sequence "a" will be generated. Then the cycle begins again with sequence ' $b$ " as prototype sequence, while sequences "a, c- $z$ " each in turn is used as target sequences to generate the list for sequence " $\mathrm{b}$ ". The cycle will continue until we generate the list for all download sequences (sequences ' $\mathrm{a}-\mathrm{z}$ "). After this is completed, the second stage of the process will be undertaken. The number of sequences that a sequence (as prototype) is predicted to detect will be sorted and ordered. The sequence that was predicted to detect the most other sequences (as targets) was selected as a probe sequence to be used in the microarray design. It was then removed from the list of sequences. All the target sequences detected by that prototype sequence were also removed from the list of sequences. This procedure was repeated until the list of sequences was empty. When two or more prototype sequences were predicted to detect the same maximal number of target sequences, one was randomly selected hence the method was non-deterministic. The process was repeated with different random seeds and the number of required probe sequences did not vary significantly while the sequences used in the microarray design could change.

\section{Application: HRV and HEV}

The described design method could be applied to any group of sequences and a minimum set of prototype regions would be determined. The group of sequences used for HRV probe design was chosen using different criteria than those used to select the HEV sequences in the HEV probe design due to differences in the available sequences for each in GenBank. At the time of this design, only eight HRV serotypes had complete genomes sequenced. These genome sequences and all complete and partial 5'UTR sequences available for HRV in GenBank were retrieved in April 2006 and a total of 150 sequences were used in the predictive modeling. A set of 26 sequences with lengths between 145 and 500 bp were predicted to provide detection of all those input sequences (Additional file 1).

Because HEV is better characterized with complete genome sequences of all 60 recognized serotypes, the design algorithm was applied to one complete genome sequence of each serotype. In addition, the design algorithm was applied to the $3 \mathrm{D}$ region. The design procedure generated 4 to 8 sequences for HEV detection using 5'UTR region, and 13 sequences were predicted to detect all HEV $3 \mathrm{D}$ regions. It was decided to use corresponding 5'UTR sequences of the 13 genomes that the $3 \mathrm{D}$ targets were selected from so that the same serotype was targeted by both target regions (Additional file 1). These 13 5'UTR regions were predicted to still provide complete and now redundant coverage.

\section{Specificity of RPM-Flu v.30/3 I chip for HRV detection and serotyping: design method I}

To assess the performance of RPM-Flu v.30/31 chip design, 34 known HRV serotype strains obtained from ATCC were tested. Of the 34 strains, 18 had corresponding 5'UTR sequences tiled on the microarray and were called prototype serotypes, which were used to verify the accuracy of the designed HRV probes. The remaining 16 strains, representing near neighbor serotypes, were selected from diverse clades based on phylogenetic classification of HRV serotypes $[18,19]$. These strains were used to investigate the capability of the microarray to detect other HRV serotypes that did not have their sequences tiled on the microarray. Overall, the selected strains covered every single clade of 101 HRV serotypes based on phylogenetic analysis of the P1-P2 regions of 5'UTR sequences [18]. One metric of the hybridization in a reference region is to divide the number of bases reported as $\mathrm{A}$, C, G, or T by the total number of bases for that region (probe length), which we refer to as the base call rate and proportionally reflects the hybridization strength or homology between the prototype and target sequences. A hybridization profile (Fig. 2A) using the base call rates clearly showed a unique pattern for each serotype. The closely related serotypes with less nucleotide divergences had similar hybridization profiles across the tiled regions, so it is possible to assign species (HRVA and HRVB) based only on the hybridization patterns. The brighter red spots (higher base call rates) along the diagonal suggested that stronger hybridizations between the tiled probes and 5 'UTRs from the prototype serotypes. It is also of note that HRV87 does not fall into either of two major clusters which agrees with other findings that it should really be classified as a HEV $[20,21]$. To validate the accuracy of the array clustering, 5'UTR of each serotype was amplified by type-specific RT-PCR and subjected to conventional sequencing. The phylogenetic tree derived from the de novo 5'UTR sequences (Additional file 2) confirms the HRVA and HRVB classification. Pair-wise sequence comparisons also indicated that the average nucleotide divergence of 5'UTR sequences between all 34 strains was $20.3 \%$. The maximal nucleotide divergences among HRVA and HRVB strains were $33.4 \%$ and $32.7 \%$, respectively. These results demonstrated that RPM-Flu v.30/31 chip is potentially capable of detecting genetically diverse HRV serotypes.

Although useful, the visual patterns are difficult to discriminate the serotypes within each species and would require generating reference patterns for all HRV serotypes. The use of sequence calls and analysis software, CIBSI [22], allows for straightforward serotype identifica- 
A
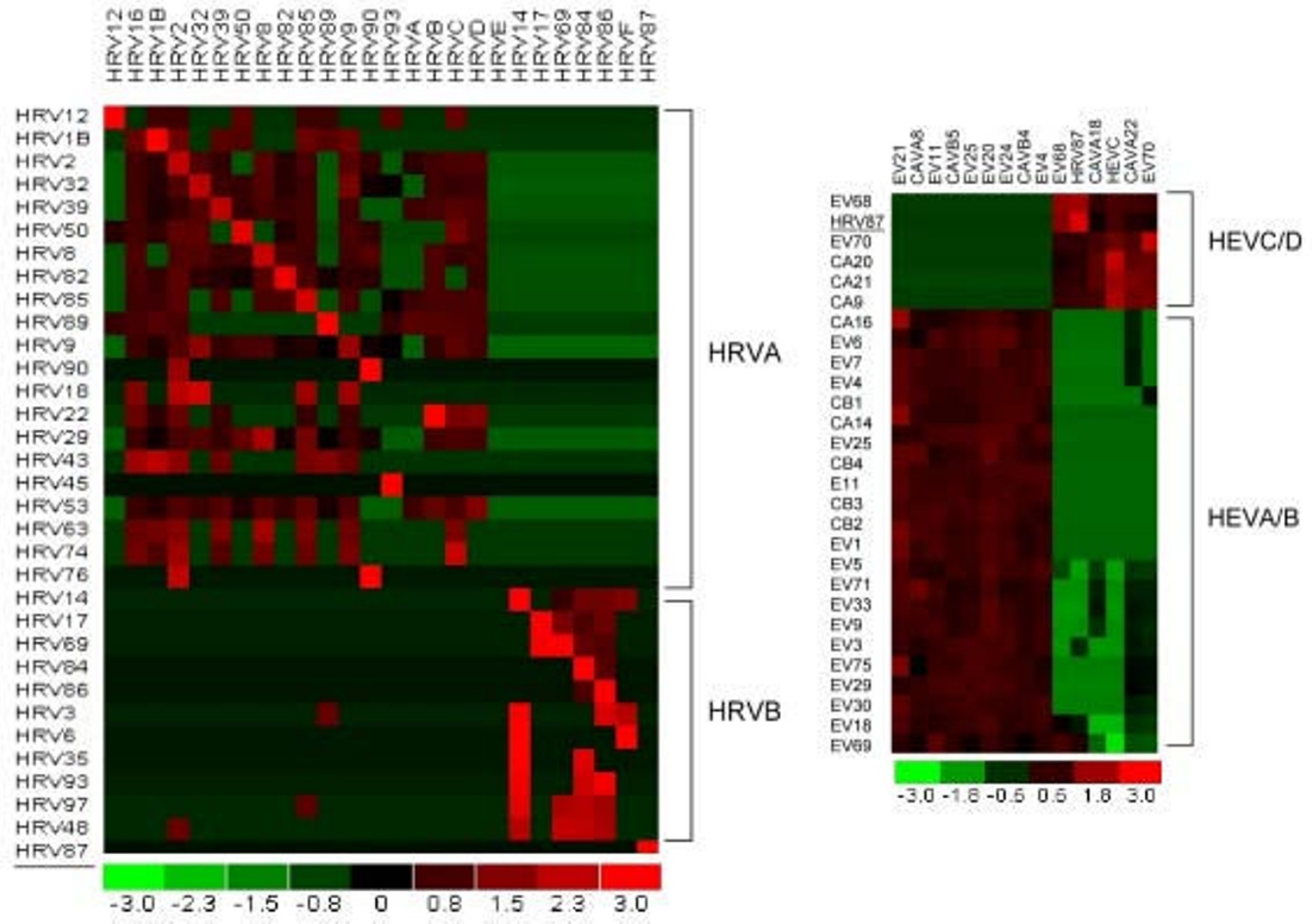

\section{Figure 2}

Hybridization profiles of HRV and HEV serotypes from RPM-Flu v.30/3 I microarrays. (A) $34 \mathrm{HRV}$ serotypes were classified into two clusters corresponding to species HRVA and HRVB; (B) 29 HEV serotypes (including HRV87) were classified into two clusters corresponding to HEVA/B and HEVC/D species. Base call rates (number of base calls/probe length in each tile) generated from viral samples (rows) and prototype probes (columns) were calculated and clustered using dChip software. Rows standardized base call rates. Positive hybridization was represented by red color. Higher base call rates were shown as brighter red colors. Negative hybridization (no base call) was represented by green color. The sample HRV87 is underlined.

tion. The sequence analysis results showed that the identifications made by RPM-Flu v.30/31 and CIBSI are consistent with the reported typing, and could clearly differentiate most of the serotypes of the HRV (Table 1). Of the $34 \mathrm{HRV}$ strains, 18 matched the selected prototype 5' UTR sequences on the RPM-Flu v.30/31 chips and showed strong hybridization signals to their corresponding prototype regions generating at least $86 \%$ base call rates. Due to the sequence homology, it is not surprising to find a noticeable and sometimes significant amount of hybridization $(20-80 \%)$ leading to base calls on prototype regions other than the correspondent prototype for most samples. Because the identification scheme used was based on comparing the sequences generated on the array to database entries, this information could be integrated into the final identification. For these samples with high base call rates, the best hit would have tens to hundreds more matched bases than the next best hit. The remaining 16 ATCC strains represent near neighbor serotypes, which 5 'UTR sequences share $>80 \%$ identifies to those of prototypes. In all but the case of HRV5, the information was sufficient to identify the correct serotypes. In these samples, the difference in the number of base calls matching in the best hit and the next best hit was more variable and on average was fewer. The confidence of the identification depends on the accuracy of the base call. Since the best hit always had at least one base that matched the resequencing results and was a mismatch for the next best hit, it was 
Table I: Identification of HRV serotypes using microarray, de novo sequencing, and in silico model of de novo sequence results.

\begin{tabular}{|c|c|c|c|c|c|}
\hline Sample name & ATCC typing & Array & de novo sequence* & in silico model & Accession Number \\
\hline VR-I I 22 & HRVI 2 & HRVI 2 & HRV I 2 (99\%) & HRVI2 & EU870449 \\
\hline VR-1366 & HRVIB & HRVIB & HRV I B (99\%) & HRVIB & EU870452 \\
\hline VR-482 & HRV2 & HRV2 & HRV2 (99\%) & HRV2 & EU870453 \\
\hline VR-I I 42 & HRV32 & HRV32 & HRV32 (100\%) & HRV32 & EU870454 \\
\hline VR-340 & HRV39 & HRV39 & HRV39 (99\%) & HRV39 & EU870455 \\
\hline VR-5I7 & HRV50 & HRV50 & HRV50 (100\%) & HRV50 & EU870457 \\
\hline VR-II92 & HRV82 & HRV82 & HRV82 (100\%) & HRV82 & EU870460 \\
\hline VR-II95 & HRV85 & HRV85 & HRV85 (99\%) & HRV85 & EU870462 \\
\hline VR-II99 & HRV89 & HRV89 & HRV89 (99\%) & HRV89 & EU870465 \\
\hline VR-III 8 & HRV8 & HRV8 & HRV8 (100\%) & HRV8 & EU870459 \\
\hline VR-489 & HRV9 & HRV9 & HRV9 (100\%) & HRV9 & EU870466 \\
\hline VR-284 & HRVI 4 & HRVI 4 & HRV I 4 (99\%) & HRVI4 & EU870450 \\
\hline VR-II 27 & HRVI7 & HRVI7 & HRVI7 (1 00\% & HRVI7 & EU87045 I \\
\hline VRV-II79 & HRV69 & HRV69 & HRV69 (100\%) & HRV69 & EU870458 \\
\hline VR-II94 & HRV84 & HRV84 & HRV84 (100\%) & HRV84 & EU87046 I \\
\hline VR-I I 96 & HRV86 & HRV86 & HRV86 (99\%) & HRV86 & EU870463 \\
\hline VR-II97 & HRV87 & HRV87 & HRV87 (99\%) & HRV87 & EU870464 \\
\hline VR-|29I & HRV90 & HRV90 & HRV90 (100\%) & HRV90 & EU870467 \\
\hline VR-II 28 & HRVI8 & HRVI8 & HRVI8 (I00\%) & HRVI8 & EU870469 \\
\hline VR-I132 & HRV22 & HRV22 & HRV22 (100\%) & HRV22 & EU870470 \\
\hline VR-II 39 & HRV29 & HRV29 & HRV29 (99\%) & HRV29 & EU87047I \\
\hline VR-II53 & HRV43 & HRV43 & HRV43 (100\%) & HRV43 & EU870474 \\
\hline VR-II84 & HRV74 & HRV74 & HRV74 (98\%) & HRV74 & EU870480 \\
\hline VRII86 & HRV76 & HRV76 & HRV76 (99\%) & HRV76 & EU87048I \\
\hline VR-483 & HRV3 & HRV3 & HRV3 (99\%) & HRV3 & EU870472 \\
\hline VR-485 & HRV5 & HRV-B & HRV5 (100\%) & HRV5 & EU870476 \\
\hline$\overline{V R-486}$ & HRV6 & $\overline{\text { HRV6 }}$ & HRV6 (99\%) & HRV6 & EU870478 \\
\hline VR-508 & HRV35 & HRV35 & HRV35 (100\%) & HRV35 & EU870473 \\
\hline VR-5I2 & HRV45 & HRV45 & HRV45 (I00\%) & HRV45 & EU870456 \\
\hline VR-I294 & HRV93 & HRV93 & HRV93 (100) & HRV93 & EU870468 \\
\hline VR- 1297 & HRV97 & HRV97 & HRV97 (93\%) & HRV97 & EU870482 \\
\hline VR-II73 & HRV63 & HRV63 & HRV62 (90\%) & HRV63 & EU870479 \\
\hline VR-5I5 & HRV48 & HRV48 & HRV48 (98\%) & HRV48 & EU870475 \\
\hline VR-II63 & HRV53 & HRV53 & HRV53 (99\%) & HRV53 & EU870477 \\
\hline
\end{tabular}

Prototype serotypes having strong hybridization signals were designated in bold characters. The strain not identified at serotype level by microarray was underlined.

*Serotype identities were made by searching 5'UTR sequences of HRV isolates against Genbank;

() indicates the highest percentage of identity to the sequence in Genbank.

possible to establish the lowest confidence level that the best hit was the correct identification. The resequencing microarray's accuracy for determining the base call has been established under a variety of conditions [23]. Using this information, there is a $.000001^{\mathrm{n}}$ that the next best hit is the most similar sequence in the database because a base is misidentified by the resequencing microarray where $\mathrm{n}$ is the number of mismatches between the next best hit and the matches for the best result. With .0001\% being the largest level of uncertainty seen for these samples, it was deemed acceptable to treat the best hit as the correct identification. For HRV5, several database sequences representing different serotypes had the same score and since no further information was available it was only possible to determine that a HRV species B was present. The 5'UTR sequences from the tested strains generated with de novo sequencing were subjected to in silico predictive modeling analysis and the result of this was used as input in the CIBSI analysis program. For the case of HRV5, the in silico model predicted a larger fraction of base calls being made than were observed in the experiment. For all other samples there was a good correspondence on base call fractions between model and experiment as expected. This leads us to suspect there was a processing error or sample degradation leading to the less accurate identification.

\section{Specificity of RPM-Flu v.30/3 I chip for HEV detection and serotyping: design method 2}

A panel of $28 \mathrm{HEV}$ serotypes, including serotypes from all four HEV species, was similarly used to validate the specificity of RPM-Flu v.30/31 for HEV detection and identification. These serotypes were originally typed based on VP1 sequences (personal communication - Steve Oberste) 
and the majority of them belonged to members of HEVB. The hybridization profile (Figure 2B) shows distinct clusters in a similar fashion to the HRV samples based on serotypes. In this case, HEVA and HEVB make up one cluster, while HEVC and HEVD (including HRV87) comprise a second cluster. This finding is consistent with the previously described clusters for HEV UTR sequences [24,25]. The redundancy of the targets that was a consequence of how they were selected is apparent in the more uniform response observed within each cluster to the various strains.

Analysis of sequences reported from RPM-Flu 30/31 array analysis indicated that two levels of identifications were obtained from 28 strains (Table 2). Serotype level identification was made for 11 of the 28 strains, in which 9 cases correlated with typing made by the VP1 genes. For example, HEV71 and Coxsackievirus A16 (CAVA16), known to cause hand-foot-mouth disease, were unambiguously recognized as HEV71 and CAVA16 respectively using RPMFlu v.30/31 and analysis program. Two strains were identified by RPM-Flu v.30/31 as HEV4 and HEV5, results in agreement with the conventional sequencing of each
5'UTR. However, the strains as provided by CDC were identified as HEV6 and CAVB3 respectively, based on the VP1 region. Specific serotypes could not be identified for the remaining sixteen samples using the sequence read generated from the array. Nevertheless these samples were easily categorized into the respective species. Due to amplification problems only a subset of strains were successfully de novo sequenced, which showed 3 - 18\% variations in 5'UTR sequences. The base call rates obtained by in silico predictions based on the de novo sequences were similar to the microarray results and the identifications agreed in all but one case.

\section{Discussion}

This study demonstrated the use of an algorithm for the design of probe sets based on an in silico predictive model [17], developed by our group, that minimized the probes needed for detection and identification of most serotypes of HRV and HEV. The potential of using resequencing microarray for simultaneous detection and identification of highly diverse respiratory pathogens, such as HRV and HEV, was also demonstrated. The conserved nature of the 5' UTR regions of HRV and HEV genomes and the capabil-

Table 2: Identification of HEV serotypes using microarray, de novo sequencing, and in silico model of de novo sequence results.

\begin{tabular}{|c|c|c|c|c|c|}
\hline Strain & VPI typing\# & Array & de novo sequence & in silico model & Accession number \\
\hline I & CAVAI 6 & CAVAI 6 & ND & ND & \\
\hline 2 & EV7I & EV7I & ND & ND & \\
\hline 3 & CAVA2I & CAVA2I & ND & ND & \\
\hline 4 & CAVB4 & CAVB4 & ND & ND & \\
\hline 5 & EV3 & EV3 & EV3(95\%) & CAVI6 & EU870485 \\
\hline 6 & EV68 & EV68 & EV68(99\%) & EV68 & EU87049I \\
\hline 7 & EV69 & EV69 & ND & ND & \\
\hline 8 & EV70 & EV70 & ND & ND & \\
\hline 9 & EV75 & EV75 & EV75(99\%) & EV75 & EU870493 \\
\hline 10 & EV4 & EV6 & EV6(93\%) & EV4 & EU870488 \\
\hline 11 & EV5 & CAVB3 & CAVB3(94\%) & CAVB3 & EU870489 \\
\hline 12 & EV30 & HEVB & EV30(95\%) & EV30 & EU870486 \\
\hline 13 & CAVAI4 & HEVA/B & ND & ND & \\
\hline 14 & EVI & HEVB & ND & ND & \\
\hline 15 & EV6 & HEVB & EV74(94\%) & HEVB & EU870490 \\
\hline 16 & EV7 & HEVB & EV30(95\%) & HEVB & EU870492 \\
\hline 17 & EVII & HEVB & ND & ND & \\
\hline 18 & EVI8 & HEVB & EV74(93\%) & HEVB & EU870483 \\
\hline 29 & EV29 & HEVB & EV80(85\%) & HEVB & EU870484 \\
\hline 20 & CAVA20 & HEVC & ND & ND & \\
\hline 21 & CAVA24 & HEVC & ND & ND & \\
\hline 22 & CAVA9 & HEVB & ND & ND & \\
\hline 23 & CAVBI & HEVB & ND & ND & \\
\hline 24 & CAVB2 & HEVB & ND & ND & \\
\hline 25 & CAVB3 & HEVB & ND & ND & \\
\hline 26 & $\mathrm{EV} 25$ & HEVB & ND & ND & \\
\hline 27 & EV33 & HEVB & CAVAI2(9I\%) & HEVB & EU870487 \\
\hline 28 & EV9 & HEVB & ND & ND & \\
\hline
\end{tabular}

\#This represents the typing provided by CDC when the samples were obtained;

*Serotype identities were made by searching 5'UTR sequences of HEV isolates against Genbank;

() indicates the highest percentage of identity to the sequence in Genbank. 
ities of the resequencing microarray allow serotype level identification of near-neighbor serotypes of HRV and HEV, when long (> 100 nucleotides) sequences are read from the array. Identifications can be still made for shorter length sequences to the species level particularly when the array has one or more such sequences derived from different probes.

The utility of the resequencing microarray is related to the target selection, the optimized prototype sequences represented on the array. In the case of RPM-Flu v.30/31, the selection of HRV targets has proved to be very robust. The 5'UTR has been shown in this study to be a good choice for serotyping HRV on RPM, as it performed similarly well on other platforms $[13,26]$. All HRV variants tested in this study could be detected and identified at least to the species level. The limited number of HRV sequences available in GenBank during the time of design of RPM-Flu v.30/31 rendered a few of the targets represented on RPM-Flu v.30/31 are shorter than 200 bp. In the past year, complete genome sequences from 46 more serotypes and another two divergent HRV'X's have been reported $[6,19,27]$. It will be worthwhile to update the design for the next generation of the chip.

In the case of HEV, the RPM-Flu v.30/31 assay identified only 11 of 28 strains tested at serotype level. Several strains not producing serotype identifications might have been indicative of assay protocol issues or probe design. The fact that the in silico model prediction was also not serotype specific indicates it was most likely a design issue. This was further confirmed by agreement in base calls made from the resequencing microarray and from conventional resequencing. Although all the strains of HEV have complete genome sequences, there are also many partial sequence submissions for each strain in GenBank that were ignored for the HEV design. A re-examination of the 5'UTR regions showed up to $14 \%$ difference in sequences grouped in the same serotype. This indicates that a redesign of the HEV prototype regions is needed where selection of a minimal set of prototype regions would be based on all available 5'UTR sequence data (complete and partial) and not a subset of genome sequences.

Comparing the identifications made from de novo sequencing to the identifications made by CDC (sources of the samples) illustrated another shortcoming of using the 5'UTR region for HEV that did not occur for HRV. Oberste et al. demonstrated that typing based upon HEV VP1 capsid gene sequences showed excellent correlation with serotype determined by classical antigenic methods [28]. Thus amplification and sequencing of the partial VP1 amino-terminal coding region has been accepted as a standard molecular typing method for HEV but such is not the case for the 5'UTR region [29-33]. Our results show that the 5'UTR region did not correlate as closely as VP1-based typing to antigenic type definitions for HEV unlike how it performed for HRV. While the 5'UTR region is sufficient to accurately identify the groupings, a design using VP1 as the probe region is needed to provide serotyping identifications that will match classical methods.

The current RPM design can detect and identify a more comprehensive set of viral and bacterial respiratory pathogens in parallel, including detailed discrimination of certain serotypes of HRV and HEV. This study showed that most shortcomings in the design were a result of not including adequate reference sequences for the initial design. The selection of VP1 and 3D regions also showed that incorporation of primer design considerations must be contemplated sooner in the design process than it has been currently done to prevent the selection of regions that cannot be used. Future development will address these limitations by reducing HEV probe redundancy and lack of coverage, by updating or confirming the HRV probes to be derived from newly available HRV sequences, and by involving primer design earlier in the overall design process.

\section{Conclusion}

A powerful feature of the expanded RPM-Flu v.30/31 resequencing pathogen microarray is that the nucleotide sequences generated from hybridization of the sample RNA/DNA and array-bound probe sets in conjunction with previously developed sequence analysis algorithm CIBSI can be easily interpreted to make serotype or strain identifications. This feature and the platform's high resolution and high throughput aspects undoubtedly have great potential for use as a diagnostic tool, and therefore, efforts are currently underway to test the utility of this array on more clinical samples. The results presented also validated the usefulness of the design methodology and it is currently being applied to assist in a new microarray application associated with other genetically diverse viruses.

\section{Methods \\ Viral strains, specimen and nucleic acid isolation}

A panel of 27 cultured enterovirus (HEV) prototype strains was purchased from Center for Disease Control and Prevention (CDC, Atlanta, GA). The prototype strains of 34 rhinoviruses (HRV) and HEV69 with known titers were purchased from the American Type Culture Collection (ATCC, Manassas, VA). Total nucleic acids were extracted from $125 \mu \mathrm{l}$ cultured samples by using the MasterPure $^{\mathrm{TM}}$ DNA purification kit (Epicentre Technologies, Madison, WI) and dissolved in $20 \mu \mathrm{l}$ of nuclease-free water. 


\section{Primer design}

All 5'UTR sequences of HRV and HEV with approximately 750 bp sizes were downloaded from GenBank. Potential PCR primer pairs that are able to amplify 600 - $700 \mathrm{bp}$ fragments from HRV and HEV were automatically selected by a perl script primer search program developed by our group using the rules described in previous publication $[10,11]$.

\section{Multiplex reverse transcription polymerase chain reaction} The multiplex reverse transcription polymerase chain reaction (RT-PCR) protocols for RPM-Flu v.30/31 were carried out as previously described [10] with the following modifications. For the RT step, primer LN was replaced by primer NLN (a random 9mer with the unique linker sequence), 1 pg each of two internal controls NAC1 and triosephosphate isomerase (TIM), and $5 \mu \mathrm{l}$ of the extracted viral nucleic acids were used. The $5 \mu$ l RT reaction product was subjected to the multiplex PCR reaction. Platinum Taq DNA polymerase (Invitrogen Life Technologies, Carlsbad, CA) was replaced by GoTaq $^{\circledR}$ DNA polymerase (Promega Corporation, Madison, WI) in the PCR reaction. Primer NL instead of primer $L$ was used with 50-150 nM each of 5'UTR primers in the multiplex PCR. The amplification reaction was carried out in a Peltier Thermal Cycler - PTC240 DNA Engine Tetrad 2 (MJ Research Inc., Reno, NV) with an initial incubation at $25^{\circ} \mathrm{C}$ for $10 \mathrm{~min}$, then preliminary denaturation at $94^{\circ} \mathrm{C}$ for $2 \mathrm{~min}$ followed by 16 cycles of $94^{\circ} \mathrm{C}$ for $30 \mathrm{~s}, 45-60^{\circ} \mathrm{C}$ for $30 \mathrm{~s}$ (incremental increase of $1^{\circ} \mathrm{C}$ per cycle), and $72^{\circ} \mathrm{C}$ for $90 \mathrm{~s}$, then 24 cycles of $94^{\circ} \mathrm{C}$ for $30 \mathrm{~s}$ and $60^{\circ} \mathrm{C}$ for 120 s.

\section{Microarray hybridization and analysis}

Microarray hybridization and processing, and the image scanning were performed according to the manufacture's recommended protocol (Affymetrix Inc., Santa Clara, CA) using a GenChip resequencing assay kit (Affymetrix) with modification as previously described [10]. After scanning, GCOS software was used to reduce the raw image (.DAT) file to a simplified file format (.CEL file) with intensities assigned to each of the corresponding probe positions. GDAS software was then used to produce nucleotide reads (A, C, G and T) or base calls, comparing the respective intensities for the sense and antisense probe sets. The sequences from base calls made for each tiled region of the resequencing microarray were exported from GDAS as the FASTA-formatted files. Base call rate refers percentage of number of base calls generated from the full length of probe in each tile.

Final pathogen identification for the RPM-Flu v.30/31 assay was performed using Computer-Implemented Biological Sequence Identifier (CIBSI) Version 2.0 software [22], an automatic pathogen identification algorithm based on nucleic acid sequence alignment, which was developed and tested in detail in previous studies $[10,11]$. The NCBI BLAST and taxonomy databases used for CIBSI analysis was downloaded in December 2007. Heat-map and clustering dendrogram was made with dChip 2005 (DNA-Chip Analyzer, http://www.dchip.org). The rows of the imported data (base call rates) were standardized and clustered. Clustering distance was 1 - correlation with average linkage, and gene ordering by cluster tightness.

\section{DNA sequencing and analysis}

5'UTR sequences were amplified from HRV- or HEV cDNA with specific primers. Amplified products were purified and sent to Macrogen USA (Gaithersburg, MD) for automated Sanger/electrophoresis-based sequencing using corresponding specific primers. Phylogenetic analysis of 5'UTR sequences was performed by using neighbor-joining method in MEGA software http://www.megasoft ware.net. All nucleotide sequences used in this study are available at GenBank (accession nos. EU870449EU870493).

\section{Authors' contributions}

ZW conceived and designed the study, performed microarray experiments, analyzed data and wrote the manuscript; AM designed microarray probes, analyzed data and wrote the manuscript; BL assisted in data analysis and preparing the manuscript; $\mathrm{CK}, \mathrm{NL}$ and $\mathrm{KB}$ performed microarray experiments; DT helped to generate heatmap; CT assisted in data analyses; DS initiated the project and helped to prepare the manuscript.

\section{Additional material}

\section{Additional file 1}

Additional Table 1. HRV and HEV target regions on RPM-Flu v.30/31 chip. This table lists sequence information of HRV and HEV tiles on RPM-Flu v.30/31 chip.

Click here for file

[http://www.biomedcentral.com/content/supplementary/14712164-9-577-S1.doc]

\section{Additional file 2}

Additional Figure 1. Phylogenetic analysis of the 5'UTR sequences of 31 HRV serotypes rooted with HRV87 showed the similar HRV clustering to microarray. de novo 5'UTR sequences of $31 \mathrm{HRV}$ serotypes tested in this study were subjected to phylogenetic analysis. Classification of HRVA and HRVB groups in the phylogenetic tree correlates with microarray clustering.

Click here for file

[http://www.biomedcentral.com/content/supplementary/14712164-9-577-S2.doc] 


\section{Acknowledgements}

The funding for this research was provided in part by the Office of Naval Research via the NRL base program. Partial support from Tessarae, LLC (Potomac Falls, VA) through cooperative research and development agreement that help make this research possible is also gratefully appreciated.

The opinions and assertions contained herein are those of the authors and are not to be construed as those of the U.S. Navy or military service at large.

\section{References}

I. Briese T, Palacios G, Kokoris M, Jabado O, Liu Z, Renwick N, Kapoor V, Casas I, Pozo F, Limberger R, et al.: Diagnostic system for rapid and sensitive differential detection of pathogens. Emerg Infect Dis 2005, I I(2):3 I0-313.

2. Ecker DJ, Sampath R, Blyn LB, Eshoo MW, Ivy C, Ecker JA, Libby B, Samant V, Sannes-Lowery KA, Melton RE, et al.: Rapid identification and strain-typing of respiratory pathogens for epidemic surveillance. Proc Natl Acad Sci USA 2005, I02(22):8012-80I7.

3. Dunbar SA: Applications of Luminex xMAP technology for rapid, high-throughput multiplexed nucleic acid detection. Clin Chim Acta 2006, 363(I-2):7I-82.

4. Quan PL, Palacios G, Jabado OJ, Conlan S, Hirschberg DL, Pozo F, Jack PJ, Cisterna D, Renwick N, Hui J, et al:: Detection of respiratory viruses and subtype identification of influenza $A$ viruses by GreeneChipResp oligonucleotide microarray. J Clin Microbiol 2007, 45(8):2359-2364.

5. Wang D, Coscoy L, Zylberberg M, Avila PC, Boushey HA, Ganem D, DeRisi JL: Microarray-based detection and genotyping of viral pathogens. Proc Natl Acad Sci USA 2002, 99(24): I 5687-I 5692

6. Kistler A, Avila PC, Rouskin S, Wang D, Ward T, Yagi S, Schnurr D, Ganem D, DeRisi JL, Boushey HA: Pan-viral screening of respiratory tract infections in adults with and without asthma reveals unexpected human coronavirus and human rhinovirus diversity. J Infect Dis 2007, 196(6):817-825.

7. Vora GJ, Meador CE, Bird MM, Bopp CA, Andreadis JD, Stenger DA: Microarray-based detection of genetic heterogeneity, antimicrobial resistance, and the viable but nonculturable state in human pathogenic Vibrio spp. Proc Natl Acad Sci USA 2005, I02(52):19109-19114.

8. Lin B, Wang Z, Vora GJ, Thornton JA, Schnur JM, Thach DC, Blaney KM, Ligler AG, Malanoski AP, Santiago J, et al.: Broad-spectrum respiratory tract pathogen identification using resequencing DNA microarrays. Genome Res 2006, I 6(4):527-535.

9. Davignon L, Walter EA, Mueller KM, Barrozo CP, Stenger DA, Lin B: Use of resequencing oligonucleotide microarrays for identification of Streptococcus pyogenes and associated antibiotic resistance determinants. J Clin Microbiol 2005, 43( I I ):5690-5695

10. Lin B, Blaney KM, Malanoski AP, Ligler AG, Schnur JM, Metzgar D, Russell $K L$, Stenger DA: Using a resequencing microarray as a multiple respiratory pathogen detection assay. J Clin Microbiol 2007, 45(2):443-452.

I I. Lin B, Malanoski AP, Wang Z, Blaney KM, Ligler AG, Rowley RK, Hanson $\mathrm{EH}$, von Rosenvinge E, Ligler FS, Kusterbeck AW, et al.: Application of broad-spectrum, sequence-based pathogen identification in an urban population. PLOS ONE 2007, 2(5):e4I9.

12. Wang Z, Daum LT, Vora GJ, Metzgar D, Walter EA, Canas LC, Malanoski AP, Lin B, Stenger DA: Identifying influenza viruses with resequencing microarrays. Emerg Infect Dis 2006, I 2(4):638-646.

13. Deffernez C, Wunderli W, Thomas Y, Yerly S, Perrin L, Kaiser L: Amplicon sequencing and improved detection of human rhinovirus in respiratory samples. J Clin Microbiol 2004, 42(7):3212-3218.

14. Loens K, leven M, Ursi D, De Laat C, Sillekens P, Oudshoorn P, Goossens $\mathrm{H}$ : Improved detection of rhinoviruses by nucleic acid sequence-based amplification after nucleotide sequence determination of the $\mathbf{5}$ ' noncoding regions of additional rhinovirus strains. J Clin Microbiol 2003, 4I(5): | 971- 1976.

15. Pallansch MA, Roos RP: Enteroviruses: polioviruses, coxsackieviruses, echoviruses, and newer enteroviruses. In Fields Virology 4th edition. Edited by: Knipe DM, Howley PM. Philadelphia: Lippincott Williams \& Wilkins; 200I.
16. Fraenkel DJ, Bardin PG, Sanderson G, Lampe F, Johnston SL, Holgate ST: Lower airways inflammation during rhinovirus colds in normal and in asthmatic subjects. Am J Respir Crit Care Med 1995, I 5 I (3 Pt I):879-886.

17. Malanoski AP, Lin B, Stenger DA: A model of base-call resolution on broad-spectrum pathogen detection resequencing DNA microarrays. Nucleic Acids Res 2008, 36( I 0):3 I 94-320I.

18. Lee WM, Kiesner C, Pappas T, Lee I, Grindle K, Jartti T, Jakiela B, Lemanske RF Jr, Shult PA, Gern JE: A diverse group of previously unrecognized human rhinoviruses are common causes of respiratory illnesses in infants. PLOS ONE 2007, 2(1 0):e966.

19. Kistler AL, Webster DR, Rouskin S, Magrini V, Credle J], Schnurr DP, Boushey HA, Mardis ER, Li H, DeRisi JL: Genome-wide diversity and selective pressure in the human rhinovirus. Virol J 2007, 4:40.

20. Blomqvist S, Savolainen C, Raman L, Roivainen M, Hovi T: Human rhinovirus 87 and enterovirus 68 represent a unique serotype with rhinovirus and enterovirus features. J Clin Microbiol 2002, 40( I I):4218-4223.

21. Oberste MS, Maher K, Pallansch MA: Evidence for frequent recombination within species human enterovirus $B$ based on complete genomic sequences of all thirty-seven serotypes. J Virol 2004, 78(2):855-867.

22. Malanoski AP, Lin B, Wang Z, Schnur JM, Stenger DA: Automated identification of multiple micro-organisms from resequencing DNA microarrays. Nucleic Acids Res 2006, 34( I 8):5300-53 I I.

23. Cutler DJ, Zwick ME, Carrasquillo MM, Yohn CT, Tobin KP, Kashuk C, Mathews DJ, Shah NA, Eichler EE, Warrington JA, et al:: Highthroughput variation detection and genotyping using microarrays. Genome Res 200I, I I(I I): I9I3-1925

24. Hyypia T, Hovi T, Knowles NJ, Stanway G: Classification of enteroviruses based on molecular and biological properties. J Gen Virol I997, 78(Pt I): I-II.

25. Oberste MS, Penaranda S, Maher K, Pallansch MA: Complete genome sequences of all members of the species Human enterovirus A. J Gen Virol 2004, 85(Pt 6): I597-I607.

26. Wang HY, Malek RL, Kwitek AE, Greene AS, Luu TV, Behbahani B, Frank B, Quackenbush J, Lee NH: Assessing unmodified 70-mer oligonucleotide probe performance on glass-slide microarrays. Genome Biol 2003, 4(I):R5.

27. Tapparel C, Junier T, Gerlach D, Cordey S, van Belle S, Perrin L, Zdobnov EM, Kaiser L: New complete genome sequences of human rhinoviruses shed light on their phylogeny and genomic features. BMC Genomics 2007, 8:224.

28. Oberste MS, Maher K, Kilpatrick DR, Pallansch MA: Molecular evolution of the human enteroviruses: correlation of serotype with VPI sequence and application to picornavirus classification. J Virol 1999, 73(3): I94I-1948.

29. Oberste MS, Maher K, Kilpatrick DR, Flemister MR, Brown BA, Pallansch MA: Typing of human enteroviruses by partial sequencing of VPI. J Clin Microbiol I999, 37(5): I 288-I 293.

30. Oberste MS, Nix WA, Maher K, Pallansch MA: Improved molecular identification of enteroviruses by RT-PCR and amplicon sequencing. J Clin Virol 2003, 26(3):375-377.

31. Caro V, Guillot S, Delpeyroux F, Crainic R: Molecular strategy for 'serotyping' of human enteroviruses. J Gen Virol 200I, 82(Pt I):79-9I.

32. Casas I, Palacios GF, Trallero G, Cisterna D, Freire MC, Tenorio A: Molecular characterization of human enteroviruses in clinical samples: comparison between VP2, VPI, and RNA polymerase regions using $R T$ nested PCR assays and direct sequencing of products. J Med Virol 200I, 65(I): I 38- I 48.

33. Manzara S, Muscillo M, La Rosa G, Marianelli C, Cattani P, Fadda G: Molecular identification and typing of enteroviruses isolated from clinical specimens. J Clin Microbiol 2002, 40( I 2):4554-4560. 